\title{
Performance Evaluation for a Hydrodynamics Application in XcalableACC PGAS Language for Accelerated Clusters
}

\author{
Akihiro Tabuchi \\ Graduate School of Systems and \\ Information Engineering, University \\ of Tsukuba \\ Ibaraki, Japan \\ tabuchi@hpcs.cs.tsukuba.ac.jp
}

\author{
Masahiro Nakao \\ RIKEN Advanced Institute for \\ Computational Science \\ Hyogo, Japan
}

\author{
Hitoshi Murai \\ RIKEN Advanced Institute for \\ Computational Science \\ Hyogo, Japan
}

\author{
Taisuke Boku \\ Center for Computational Sciences, \\ University of Tsukuba \\ Graduate School of Systems and \\ Information Engineering, University \\ of Tsukuba \\ Ibaraki, Japan
}

\author{
Mitsuhisa Sato \\ RIKEN Advanced Institute for \\ Computational Science \\ Hyogo, Japan \\ Graduate School of Systems and \\ Information Engineering, University \\ of Tsukuba \\ Ibaraki, Japan
}

\begin{abstract}
Clusters equipped with accelerators such as GPUs and MICs are used widely. To use these clusters, programmers write programs for their applications by combining MPI with one of the accelerator programming models such as CUDA and OpenACC. The accelerator programming component is becoming easier because of a directive-based OpenACC, but complex distributed-memory programming using MPI means that programming is still difficult. In order to simplify the programming process, XcalableACC

(XACC) has been proposed as an "orthogonal" integration of the PGAS language XcalableMP (XMP) and OpenACC. XACC provides the original XMP and OpenACC features, as well as their extensions for communication between accelerator memories. In this study, we implemented a hydrodynamics mini-application CloverLeaf in XACC and evaluated the usability of XACC in terms of it performance and productivity. According to the performance evaluation, the XACC version achieved $87-95 \%$ of the performance of the MPI+CUDA version and $93-101 \%$ of the MPI+OpenACC version with strong scaling, and $88-91 \%$ of the MPI+CUDA version and $94-97 \%$ of the MPI+OpenACC version with weak scaling. In particular, the halo exchange time was better with XACC than MPI+OpenACC in some cases because the Omni XACC runtime is written in MPI and CUDA, and it is well tuned. The productivity evaluation showed that the application could be implemented after small changes compared with the serial version. These results demonstrate that XACC is a practical programming language for science applications.
\end{abstract}

Permission to make digital or hard copies of part or all of this work for personal or classroom use is granted without fee provided that copies are not made or distributed for profit or commercial advantage and that copies bear this notice and the full citation on the first page. Copyrights for third-party components of this work must be honored. For all other uses, contact the owner/author(s).

HPC Asia 2018 WS, January 31, 2018, Chiyoda, Tokyo, Japan

(C) 2018 Copyright held by the owner/author(s).

ACM ISBN 978-1-4503-6347-1/18/01.

https://doi.org/10.1145/3176364.3176365

\section{CCS CONCEPTS}

- Applied computing $\rightarrow$ Physics; • Software and its engineering $\rightarrow$ Distributed programming languages; • Computer systems organization $\rightarrow$ Heterogeneous (hybrid) systems;

\section{KEYWORDS}

Accelerator, Cluster, GPU, Hydrodynamics, OpenACC, PGAS, XcalableMP

\section{ACM Reference Format:}

Akihiro Tabuchi, Masahiro Nakao, Hitoshi Murai, Taisuke Boku, and Mitsuhisa Sato. 2018. Performance Evaluation for a Hydrodynamics Application in XcalableACC PGAS Language for Accelerated Clusters. In HPC Asia 2018 WS: Workshops of HPC Asia 2018, January 31, 2018, Chiyoda, Tokyo, Japan. ACM, New York, NY, USA, 10 pages.

https://doi.org/10.1145/3176364.3176365

\section{INTRODUCTION}

In the field of high-performance computing, clusters equipped with accelerators such as graphics processing units (GPUs) and many integrated cores (MICs) are used widely to improve computing performance. CUDA and OpenCL are employed widely for accelerator programming. It is necessary to describe codes explicitly for the device kernel and memory, so they can exploit the performance of the accelerator. Directive-based programming models such as OpenACC [11] and OpenMP 4.0 or later are well known in accelerator programming, where these models allow programmers to readily offload codes to accelerators better than conventional CUDA or OpenCL with fairly good performance.

For accelerated clusters, MPI is still used frequently for distributed-memory programming on multiple nodes, where programmers can describe applications with MPI and an accelerator programming method. However, this approach reduces productivity because it requires data and work distribution manually as well as communication using complex MPI functions. Thus, a partitioned global address space (PGAS) language XcalableMP (XMP) [19] has 
been proposed as an alternative to MPI. Furthermore, a PGAS language XcalableACC (XACC) has also been proposed as an "orthogonal" integration of XMP and OpenACC [15]. The characteristic feature of XACC is communication between accelerators across nodes, which is not supported by XMP or OpenACC. Previously, we reported that XACC provides good performance and high productivity for some benchmark programs $[10,16]$. However, in order to assess the usability of XACC, more evaluations are required with various types of practical applications. Thus, in this study, we implemented a hydrodynamics mini-application called CloverLeaf [4] in XACC and evaluated its performance and productivity.

The main contributions of this study are summarized as follows.

- We implemented a mini-application called CloverLeaf in XACC. This is the first XMP or XACC application to use a staggered grid arrangement [3], which is often used for flow simulation on a structured grid.

- We evaluated the implementation in terms of its performance and productivity.

- We proposed XMP/XACC syntax extensions to improve the implementation of CloverLeaf.

The remainder of this paper is organized as follows. Section 2 introduces related research and Section 3 describes XACC as well as some sample code. Section 4 explain CloverLeaf and its implementation in XACC. We present the performance and productivity evaluations in Section 5. Finally, we give our conclusions in Section 6.

\section{RELATED WORK}

Some application codes have been implemented with XMP. Thus, Tsugane et al. implemented and evaluated a nuclear fusion simulation code with XMP [17], where they proposed a hybrid-view model that used both a global-view model based on directives and a local-view model based on the coarray feature [13] in the application. The global-view model was used for static grid space data and the local-view mode was used for dynamic particle data. This method increased the readability with only a slight degradation in performance.

We proposed an XACC global-view model for accelerated clusters [10], where we described the syntax of the XACC global-view model as well as evaluating the performance and productivity using the Himeno benchmark. In particular, the XACC communication library used tightly coupled accelerators (TCA), which is a proprietary interconnect for accelerators that provides low-latency communication [2]. This method achieved better performance than OpenACC + MPI/InfiniBand with the Himeno benchmark, and we demonstrated that the XACC high-level communication description has the capacity to be hardware independent and facilitate high-performance programming. Moreover, we proposed the XACC local-view model [16], which employs a coarray feature for onesided communication on accelerator memories. We implemented both global-view and local-view models using MPI, and evaluated their performance and productivity with the Himeno benchmark and NAS Parallel Benchmarks CG benchmark, as well as discussing their proper use. Moreover, we evaluated XACC by using a lattice quantum chromodynamics mini-application, and the XACC version was described with only small changes compared with the serial version, where it achieved almost the same performance as the MPI+ OpenACC version and comparable performance with the MPI+CUDA version [9].

CloverLeaf has been implemented for accelerators using CUDA, OpenCL, and OpenACC $[4,6]$. In these studies, they describe each CloverLeaf implementation in detail and analyze their performance, productivity, and portability. OpenACC is a viable accelerator programming model that improves productivity and achieves better performance than poorly optimized OpenCL and CUDA.

Mallinson et al. implemented CloverLeaf using other PGAS models comprising coarray Fortran (CAF) and OpenSHMEM, and compared them with the MPI implementation [7]. They determined the performance of CloverLeaf with some halo exchange methods (e.g., putting strided data directly and putting packed data). They demonstrated that the performance of OpenSHMEM was portable with both Cray and SGI systems, and library-based OpenSHMEM performed better than language-based CAF. In this study, we also used a PGAS language called XACC, but our approach employed a global-view programming model rather than the local-view programming model used by CAF and OpenSHMEM.

\section{XCALABLEACC}

$\mathrm{XACC}[15]$ is a PGAS language that integrates the existing PGAS language XMP and OpenACC. XACC is an extension of C and Fortran, which employs XMP's distributed-memory programming for cluster nodes and OpenACC's accelerator programming. In addition, XACC provides communication for data on accelerator memories across nodes.

\subsection{XcalableMP programming}

XACC employs XMP features for distributed-memory programming on accelerated clusters. XMP extends $\mathrm{C}$ and Fortran for parallel programming on distributed-memory systems. XMP was designed by the XMP Specification Working Group of the PC Cluster Consortium [12] in Japan and it provides two programming models: the global-view model describes the actions of all nodes by adding directives to serial codes, and the local-view model describes the actions of each node, such as MPI programming and communication with other nodes using a coarray. We only used the global-view model in this study, and thus we provide its description.

The global-view model is a directive-based programming model, which was influenced by high performance Fortran (HPF) [5]. We can develop distributed-memory programs by adding XMP directives to the serial codes. HPF provides directives for only data distribution, whereas XMP provides directives for data distribution, work mapping, communication, and synchronization; therefore, XMP makes it easier to tune codes than HPF. Figure 1 shows an example of programming in the XMP global-view model. In the global-view model, a node is an execution unit of XMP, where it has a similar role to a process in MPI. A nodes directive defines a set of nodes. A template directive defines a template, which is a virtual array that represents the index space. XMP uses a template for data and work mapping. The template can be defined with undefined size and a template_fix directive defines the size later. A distribute directive distributes a template on a 


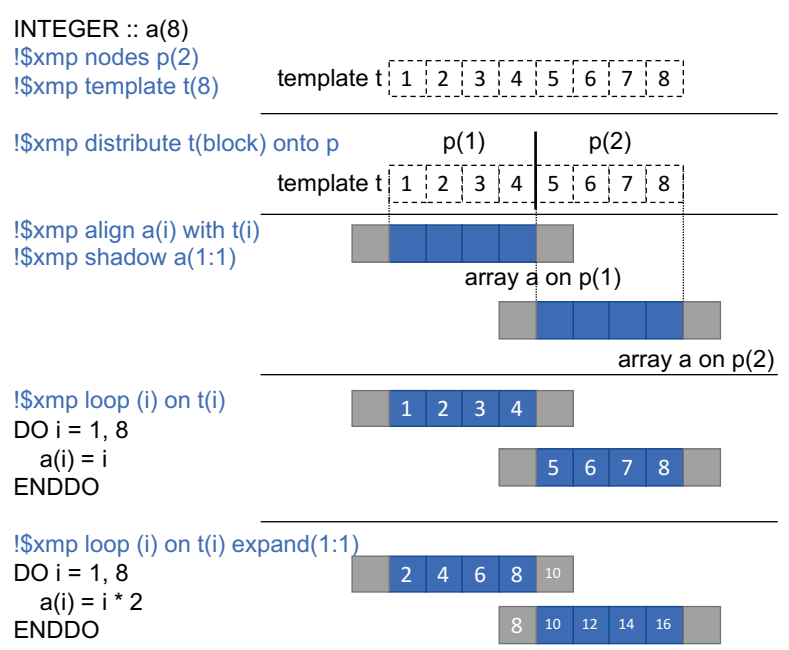

Figure 1: Example of XMP global-view model programming.

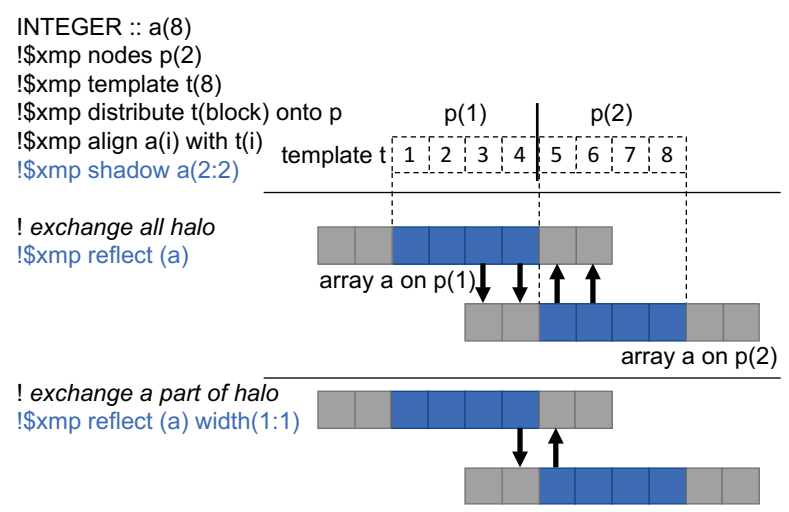

Figure 2: Example showing the use of shadow and reflect directives.

node set in a specific manner (block, cyclic, gblock, etc.). The gblock manner is shortened to a "general block" and programmers can specify blocksizes for each node explicitly in the form of "gblock(blocksize-array)." A align directive distributes arrays as well as the distributed template. Moreover, a shadow directive defines a shadow (halo or ghost) region to the distributed array. The width can be specified in the form of "(lower-width: upper-width)." A loop directive distributes the following loop iterations as well as the template. Usually, a loop range does not exceed the local range of template. However, some loops must process a larger range than the local template range, and thus XMP provides an expand clause (introduced from the XMP specification version 1.3) to expand the loop range outside the local range.

XMP provides some directives for communication. The most characteristic XMP feature is a reflect directive for halo exchange in stencil computations. Figure 2 shows an example of the reflect directive. The array $a$ is distributed on two nodes and it has a shadow with a width of two. The reflect directive updates the shadow region with the values for the actual region on neighbor

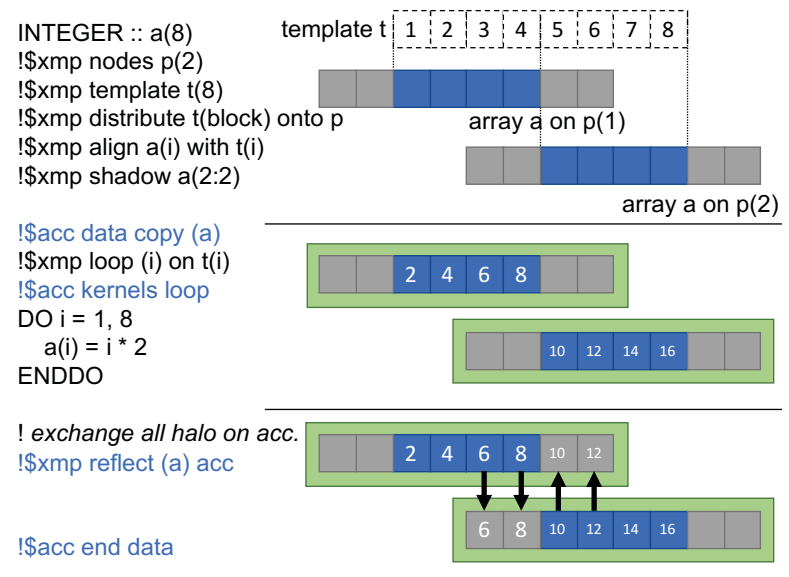

Figure 3: Example showing XACC global-view model programming.

nodes. The width clause can specify the update widths, and the directive updates all shadow regions if there is no width clause.

\subsection{XcalableACC programming}

XACC uses OpenACC features for accelerator programming. Ope$\mathrm{nACC}$ is a directive-based accelerator programming model for $\mathrm{C}$, $\mathrm{C}++$, and Fortran. Figure 3 shows an example of the XACC globalview model programming. A data directive specifies data allocation to the accelerator memory. If distributed arrays are specified in the data directive, then each node allocates the assigned parts of the arrays to its own accelerator memory. A kernels loop directive offloads the following loop to an accelerator. Similar to the data directive, each node offloads the assigned parts of the loop to its own accelerator.

XACC provides the acc clause to all XMP communication directives for communicating data in the accelerator memory. For example, using the reflect directive with the acc clause updates the shadow region of the distributed array in the accelerator memory but it does not affect anything in the host memory.

\section{CLOVERLEAF IMPLEMENTATION WITH XCALABLEACC}

CloverLeaf is a mini-application, which was developed by the UK Atomic Weapons Establishment and the University of Warwick. The code was published on GitHub by the UK Mini-App Consortium [18] and it is also part of the Mantevo project [8]. This application solves compressible Euler equations on a two-dimensional Cartesian grid, where it uses explicit finite volume methods and solves the equations with second-order accuracy. The physical quantities are arranged on a staggered grid, where some quantities are at the centers of cells (e.g., pressure) and others are at the corners of cells (e.g., velocity), as shown in Figure 4. The application repeats the following two steps. First, the Lagrangian step advances the time step by using a predictor-corrector scheme. Second, the advection step remaps the cells moved in the first step with fluid velocity. 


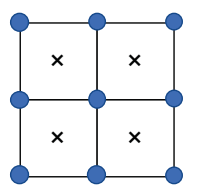

Quantity at the corner of cell

$\times$ Quantity at the center of cell

Figure 4: Staggered grid arrangement in CloverLeaf.

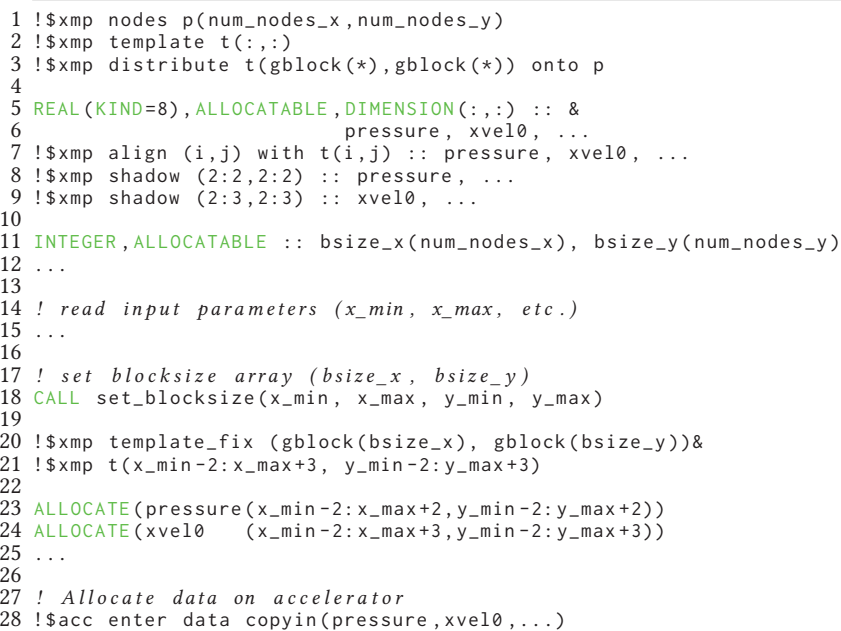

Figure 5: CloverLeaf code with XACC (definition and allocation).

In the following, we provide an overview of the implementation of CloverLeaf with XACC. We employ the XACC global-view model because this model is suitable for describing two-dimensional block decomposition and halo exchange communication in CloverLeaf. Figure 5 shows the array definition and allocation parts of the CloverLeaf code in XACC. Line 1 defines a two-dimensional nodes $p$ (num_nodes_x, num_nodes_y). L 2 defines a non-fixed size template $t(:,:)$ and line 3 distributes $t$ onto $p$ in a gblock-manner without blocksize-arrays. After reading the parameters $\left(x \_\min , x_{-}\right.$max , $y_{-} \min , y_{-} \max$, etc.) from the input file "clover.in," lines 20 and 21 specify the manner of template distribution and the size. The size is $\left(x \_\min -2: x \_\max +3, y \_\min -2: y \_\max +3\right)$, which is the same size as the largest array (the details are provided later). The outer region is used for the boundary condition; therefore, we use the manner of gblock to distribute the subregions ( $x_{-}$min : $x_{-} \max , y_{-}$min : $y \_\max$ ) evenly to the nodes. For the gblock distribution, the blocksize of the lowest node is two more than that of the others and the blocksize of uppermost node is three more than that of the others. For example, if $x \_$min $=1, x \_$max $=100$, bsize $\_x=(/ 27,25,25,28 /)$.

Quantity arrays such as pressure and xvel0 are defined as allocatable two-dimensional arrays and they are allocated after reading the parameters. The array size for quantities at the cell center is (x_min-2: $\left.x_{-} \max +2, y_{-} \min -2: y_{-} \max +2\right)$, which contains an outer halo region with a width of two for storing the boundary condition. In addition, the array size for quantities at the cell corner

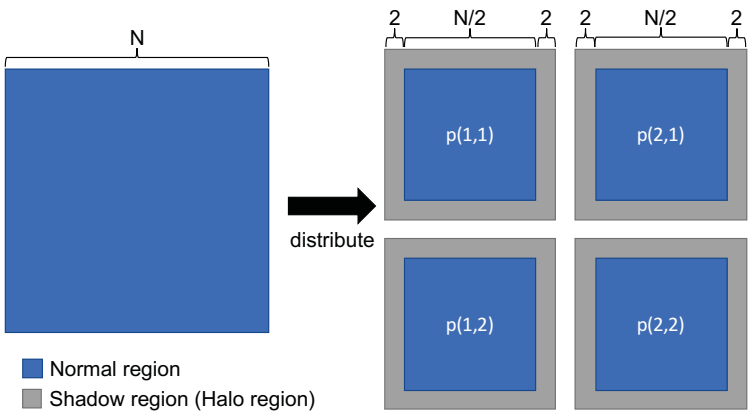

Figure 6: Array distribution for the quantity at the cell center in XMP.

is $\left(x \_\min -2: x \_\max +3, y \_\min -2: y_{-} \max +3\right)$, which has one more element in each dimension than that for the quantities at the cell center. This is because the number of cell corners is $((m+1) \times(n+1))$ if the number of cell centers is $(m \times n)$. We refer to the extended part of the arrays as the "extra region."

Line 7 specifies that quantify arrays are distributed-arrays, and lines 8 and 9 specify the shadow region. Figure 6 shows the array distribution for the quantities at the cell centers. The figure on the left-hand side represents an array in serial code, where the size of the $\mathrm{X}$ dimension is $N=\left(x \_\max +2\right)-\left(x \_\min -2\right)+1$. The figure on the right-hand side represents the array distributed on $2 \times 2$ nodes. Each distributed array has a shadow region with a width of two on the lower and upper side for halo exchange; hence, the array size in the $\mathrm{X}$ dimension is $N / 2+4$. Figure 7 shows the array distribution for the quantities at the cell corners. The figure on the left-hand side represents an array in serial code, which has an extra region, where the array size in the $\mathrm{X}$ dimension is $N+1$. The figure on the right-hand side represents the array distributed on $2 \times 2$ nodes and it is slightly complicated. The distributed array has a shadow region with a width of two on the lower side and three on the upper side. The only upper shadow width is three because the extra regions (dark gray regions in the figure) are required when the array is divided. The extra region by the shadow region is treated as a normal region during computations and it does not require updating by halo exchange. The distributed array size in the $\mathrm{X}$ dimension is $N / 2+5$ on $\mathrm{p}(1,1), \mathrm{p}(1,2)$ and $N / 2+6$ on $\mathrm{p}(2,1), \mathrm{p}(2,2)$. Finally, in line 28 , the OpenACC enter data directive also allocates arrays to the accelerator memory in advance to avoid frequent memory allocation and deallocation.

Next, we describe the distribution and offloading computation process. Similar to data distribution, we distribute double-nested loops on a two-dimensional node set and we also offload it to the accelerator on each node. Loop parallelization on the accelerators is the same as that found in the existing CloverLeaf implementation in MPI+OpenACC. Figure 8 shows the parts of the accelerate_kernel subroutine in XACC. Some parameters of the subroutines are distributed arrays (e.g., pressure, xvel0); therefore, line 812 also defines the array distributions in the subroutines. In line 15 , the data present directive states that specified arrays are already present in the accelerator memory. Most of the computations in CloverLeaf are constructed as double-nested loops. In lines 17 


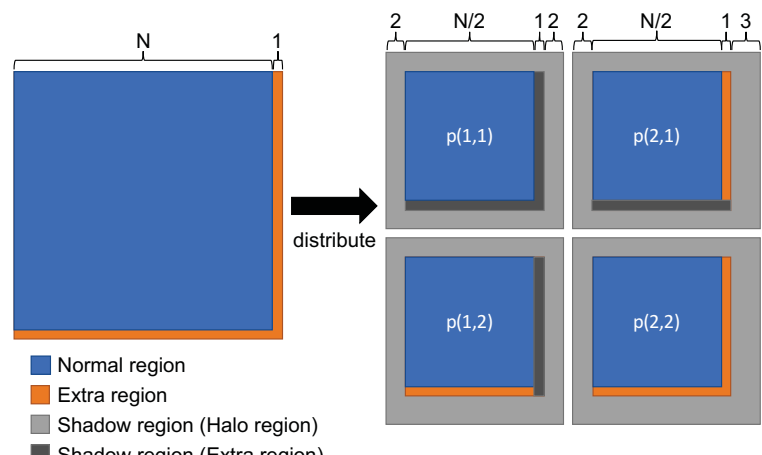

Figure 7: Array distribution for the quantity at the cell corner in XMP.

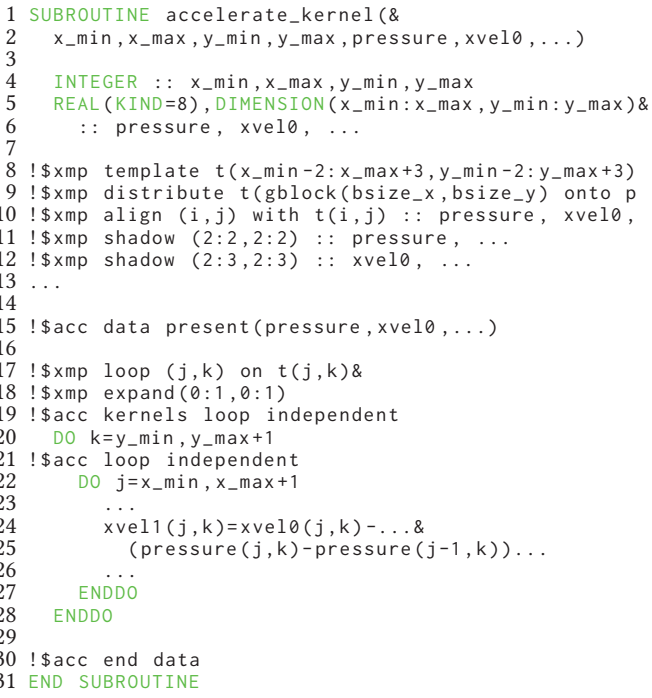

Figure 8: CloverLeaf code with XACC (computation).

and 18, the loop directives specify the work distribution for the nested loops. In these loops, some loop sections are slightly larger than others in order to process the extra regions for the cell corner quantities. In these cases, we add an expand clause to the loop directive in order to expand the loop section on each node. Lines 19 and 21 offload the distributed loops to the accelerator on each node. The MPI+OpenACC version specifies the offloading region containing multiple nested loops using the kernels directive. The XACC specification allows us to use the XMP loop directive inside the kernels region unless the loop range is changed in the region, but the current XACC compiler does not support this option. Thus, we use the kernels loop directive for each nested loop.

The main type of communication in CloverLeaf is halo exchange. The application updates the halo with a depth of one or two. MPI+OpenACC version packs the halo data on the accelerator and exchanges it via the host memory. XACC version uses the reflect directive with the acc clause and width clause to specify the depth.
Table 1: Node configuration for HA-PACS/TCA.

\begin{tabular}{l|l}
\hline \hline CPU & Intel Xeon-E5 2680v2 2.8 GHz $\times 2$ Socket \\
Memory & DDR3 1866 $\mathrm{MHz} \times 4$ channel, 128 GB \\
GPU & NVIDIA Tesla K20X × $4(\mathrm{GDDR} 56 \mathrm{~GB})$ \\
\hline Interconnect & InfiniBand: \\
& Mellanox Connect-X3 FDR $\times 1$ \\
\hline Compiler & PGI 16.10, CUDA 8.0 \\
& MVAPICH2 2.2 \\
& Omni Compiler $1.2 .1+$ extension \\
\hline
\end{tabular}

For arrays of quantities at the cell center, the XACC version updates an appropriate halo region by specifying width (depth:depth)); however, for arrays with quantities at the cell corner, we need to specify width(depth:depth+1) because it cannot exclude the innermost shadow element, and this leads to unnecessary updating.

\section{EVALUATION}

In the evaluation, we compared the performance and productivity of the application using the MPI+CUDA, MPI+OpenACC, and $\mathrm{XACC}$ versions. The official MPI+CUDA and MPI+OpenACC versions were obtained from the GitHub repository ${ }^{1}$ and we used them with some code modifications. In the MPI+CUDA version, we changed the thread-block size to 128 from 256 and the pack/unpack/copy order in halo exchange to improve the performance and cleaned up the code. In the MPI+OpenACC version, we changed the communication order for halo exchange so it was the same as that in the MPI+CUDA version and we cleaned up the code.

\subsection{Performance}

We measured the performance using the HA-PACS/TCA [1] at the Center for Computational Sciences, University of Tsukuba, Japan. The evaluation environment is shown in Table 1. We used the PGI compiler as the OpenACC implementation and MVAPICH2 as the MPI implementation. We also used the Omni XACC compiler [10, 16], which is a source-to-source XACC compiler based on the Omni compiler infrastructure [14], where Figure 9 shows the compilation flow. An input XACC code was translated into an OpenACC code with XACC runtime calls by using the XACC translator. The OpenACC code was compiled using a general OpenACC compiler (we used PGI compiler in this study) and linked with the Omni XACC runtime library. The XACC runtime library was implemented using MPI and CUDA for NVIDIA GPU clusters.

We used up to 16 compute nodes and placed four processes on each node, and thus we used up to 64 processes. We selected two problem sizes, i.e., $960^{2}$ and $3840^{2}$ cells, where we set the number of time steps to 1000 and measured the execution time with strong and weak scaling.

Figures 10 and 11 show the execution times for $960^{2}$ and $3840^{2}$ cells with strong scaling. The figures on the left show the overall time and the figures on the right show the halo exchange time. With $960^{2}$ cells, the performance levels for the MPI+OpenACC and

\footnotetext{
${ }^{1}$ https://github.com/UK-MAC/CloverLeaf
} 


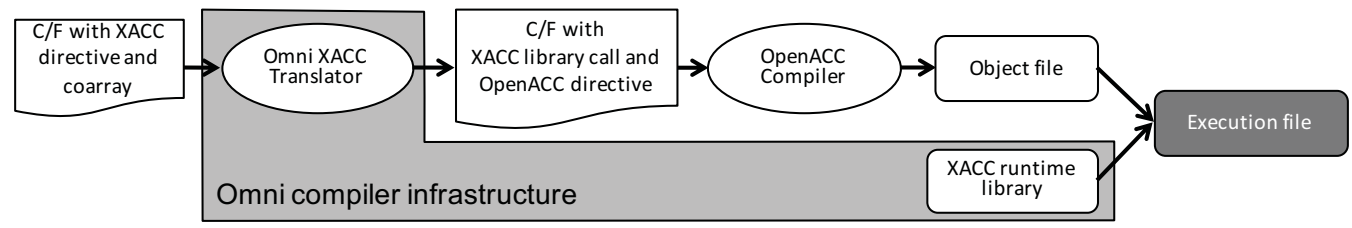

Figure 9: Compilation flow for the Omni XACC compiler.

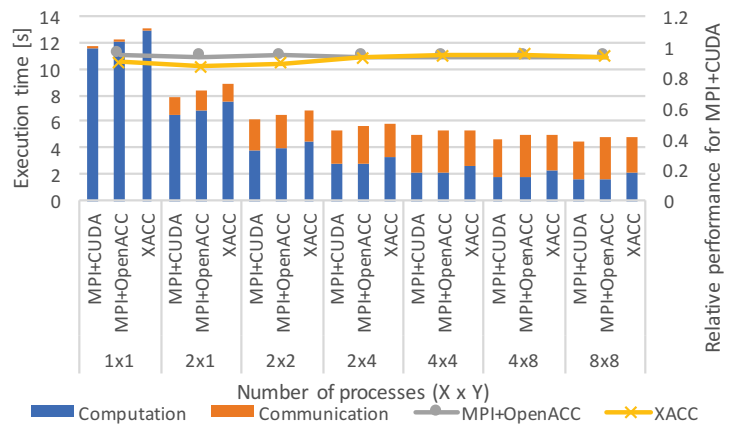

(a) Overall time

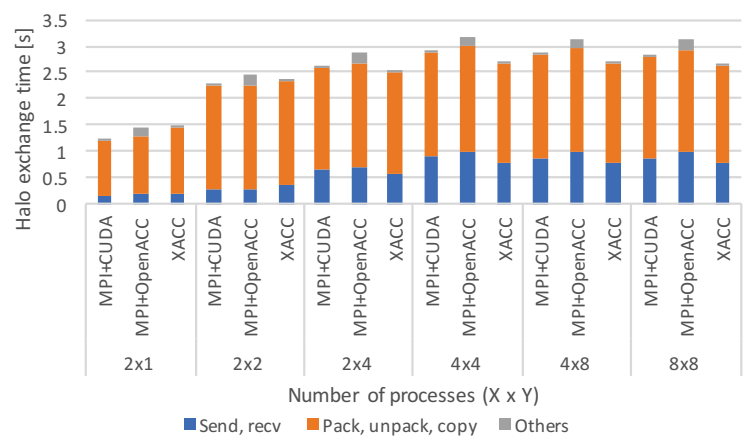

(b) Halo exchange time

Figure 10: Execution time for $960^{2}$ cells with strong scaling.

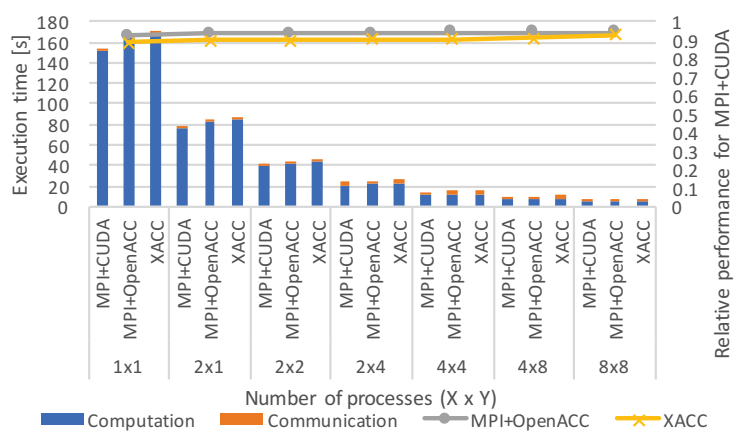

(a) Overall time

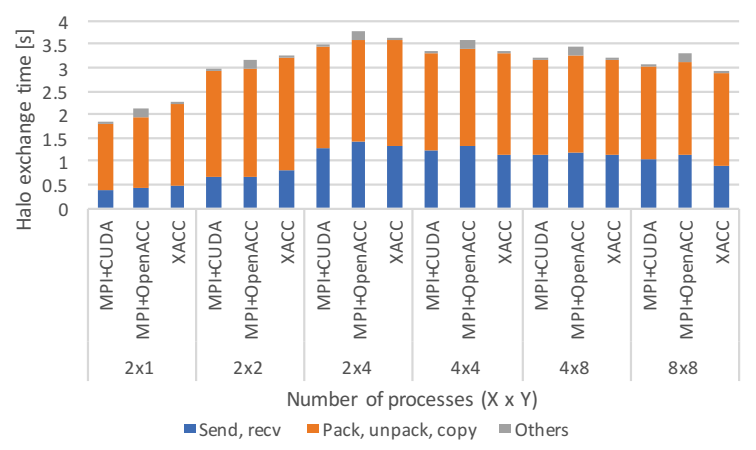

(b) Halo exchange time

Figure 11: Execution time for $3840^{2}$ cells with strong scaling.

XACC versions were $93-95 \%$ and $87-95 \%$ compared with the MPI+CUDA version, respectively, and with $3840^{2}$ cells, they were 92$94 \%$ and $89-93 \%$, respectively.

The computational time required by the MPI+OpenACC version was up to 1.05 and 1.09 times longer than that by MPI+CUDA with $960^{2}$ and $3840^{2}$, respectively, because of the difference in performance between CUDA and OpenACC. The XACC version also uses OpenACC, and thus the computational time required by the XACC version should have been the same as that by MPI+OpenACC, but it was actually up to 1.24 and 1.12 times longer than that by MPI+OpenACC with $960^{2}$ and $3840^{2}$, respectively. The longer computational time with the XACC version was due to code transformation by the XACC translator. Figure 12 shows the translated XACC code shown in Figure 8. In the XACC code shown in Figure 8, the subroutine parameters such as pressure are explicit-shape arrays and they are defined as distributed-array according to the align directive. In this case, the compiler can understand all of the array sizes based on $x_{-} \min , x_{-} \max , y_{-} \min$, and $y_{-} \max$, and optimize the offset calculations for array accesses and register utilization in GPU kernels. By contrast, in the translated code, the parameters 


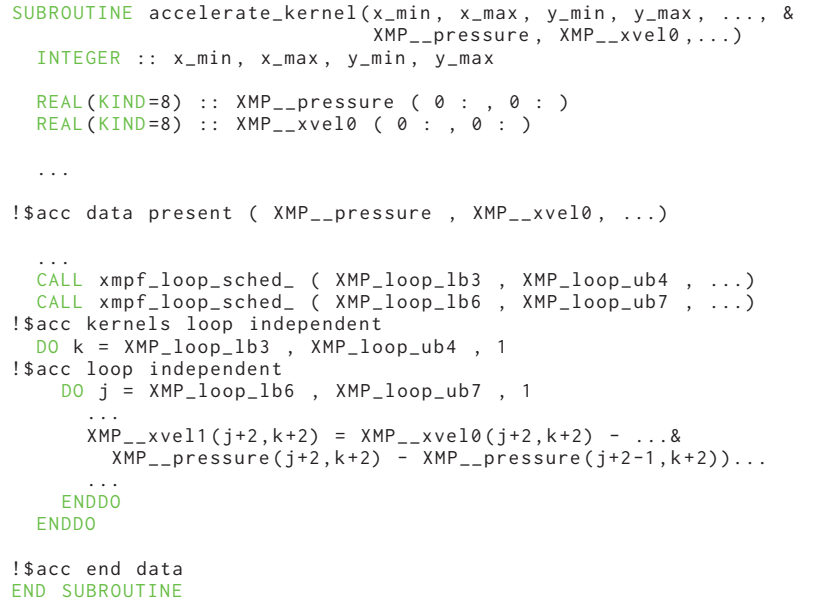

Figure 12: Translatation of the CloverLeaf XACC code in Figure 8.

are defined as assumed-shape arrays because the Omni runtime library determines the array sizes dynamically. In this case, the array sizes are undefined at the compile time and the compiler cannot optimize the offset calculations, which increases the register utilization by GPU kernels and decreases the number of concurrent execution threads.

The time required by MPI+CUDA and the XACC version for halo exchange was almost the same because the runtime of the reflect directive is written in MPI+CUDA and well tuned. Figure 13 shows the amount of halo communication required per iteration on each node with strong scaling. The amount of communication was larger with the XACC version than the other methods because the XACC version updates a slightly larger region for the cell corner quantity arrays. This difference increases the communication time when the number of nodes is small, but the effect is slight as the number of nodes increases. The halo exchange time required by the MPI+OpenACC version was slightly longer because the packing and unpacking kernels written in OpenACC are slower than those with CUDA in the MPI+CUDA and XACC versions. Moreover, the others time required by the MPI+OpenACC version was slightly longer than compared with the other methods. An overhead is incurred due to the OpenACC data present directive, which tells the compiler that the data already exists in the accelerator memory. The MPI+OpenACC version specifies the targeting array and eight buffers (four directions $\times$ send/receive) for the data present directive. By contrast, the XACC version specifies only the targeting array for the OpenACC host_data use_device directive to pass the accelerator memory address to the XACC runtime function because the XACC runtime internally manages the buffers for halo exchange.

In addition, the execution times for $960^{2}$ and $3840^{2}$ cells with weak scaling are shown in Figures 14 and 15, respectively. With $960^{2}$ cells, the performance levels of the MPI+OpenACC and XACC versions were $94-95 \%$ and $88-91 \%$ compared with the MPI+CUDA version, respectively, and with $3840^{2}$ cells, they were $92 \%$ and $89 \%$,
Table 2: SLOC and DSLOC in CloverLeaf

\begin{tabular}{l|r|r|r|r|r}
\hline \hline Code version & SLOC & \multicolumn{4}{|c}{ DSLOC } \\
\hline Serial & 3043 & Modify & Add (code) & Add (directive) & Delete \\
\cline { 3 - 6 } MPI+CUDA & 5397 & - & - & - & - \\
MPI+OpenACC & 4042 & 20 & 713 & 286 & 0 \\
XACC & 3746 & 14 & 84 & 619 & 0 \\
\hline
\end{tabular}

respectively. For all versions and problem sizes, the execution time changed little as the number of processes increased, and thus CloverLeaf exhibited good weak scalability.

The trend in the computational time was the same as that with strong scaling. The computation time required by the MPI+OpenACC version was up to 1.09 times longer than that by the MPI+CUDA version, and the computational time required by the XACC version was up to 1.07 times longer than that by the MPI+OpenACC version.

The trend in the halo exchange time with $960^{2}$ was the same as that with strong scaling. However, with $3840^{2}$, the send/receive time was longer for the XACC version than the other methods with all node configurations. Figure 16 shows the amount of halo communication per iteration on each node with weak scaling. The amount of communication required by the XACC version was larger than that with $960^{2}$ cells and the effect was significant.

\subsection{Productivity}

In MPI+CUDA or MPI+OpenACC, programmers need to describe data as well as the work distribution and communication using MPI function calls with many arguments, such as a buffer, element type, the number of elements, tag, and status. In addition, it is necessary to copy data between the host and device memories for communication on the accelerator device memory. Moreover, we may need to pack and unpack data during communication with a multidimensional array such as halo exchange. This complexity causes difficulties with the description and bugs. By contrast, XACC allows programmers to describe codes simply by slightly modifying and adding directives to the serial versions of codes. In particular, XACC provides some directives for typical types of communication such as halo exchange and the acc clause for communication on the device memory. Thus, we can describe codes for accelerated clusters in high-level syntax without considering the array size, loop range, communication buffer, pack/unpack kernels, etc.

In the quantitative comparison, we counted the source lines of code (SLOC) and the delta source lines of code (DSLOC) from the serial versions, as shown in Table 2. For DSLOC, "Add (code)" and "Add (directive)" represent the addition of non-directive codes such as Fortran and the addition of directives, respectively. For MPI+CUDA, the table only shows SLOC because the CUDA code is quite different from the serial Fortran code. The SLOCs for the MPI+CUDA, MPI+OpenACC, and XACC versions were 1.77, 1.33, and 1.23 times larger than that for the serial version, i.e., the XACC version was the smallest of the three parallel versions. The difference in SLOCs between the MPI+OpenACC and XACC versions was not very large, but difference in DSLOCs for the MPI+OpenACC was due mostly to the addition of non-directive code whereas that for XACC was largely due to the addition of directive code. This indicates that the 


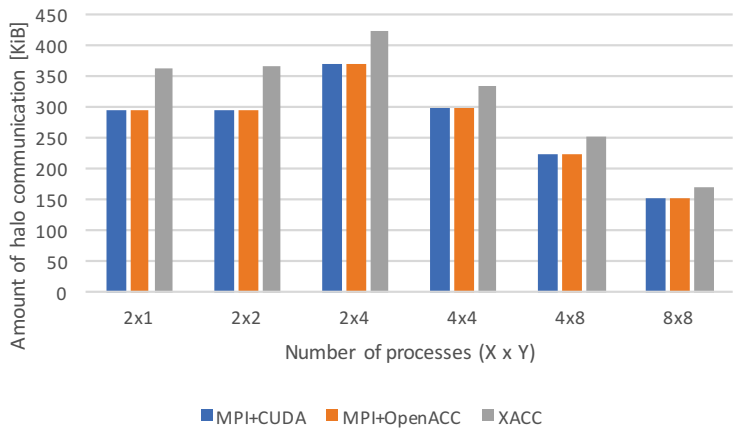

(a) $960^{2}$ cells

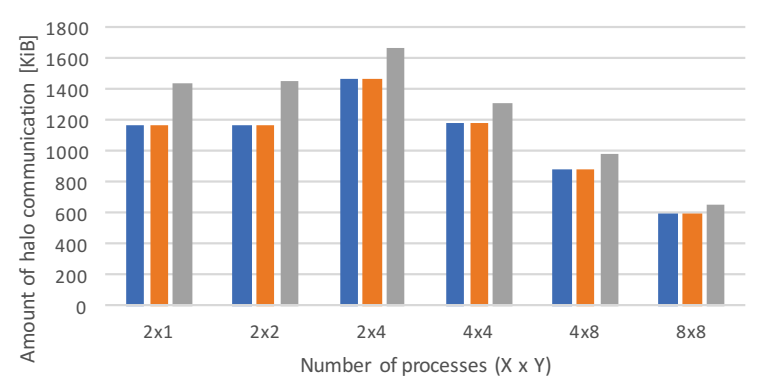

-MPI+CUDA $=\mathrm{MPI+OpenACC}=\mathrm{XACC}$

(b) $3840^{2}$ cells

Figure 13: Halo communication amount per iteration on each node with strong scaling.

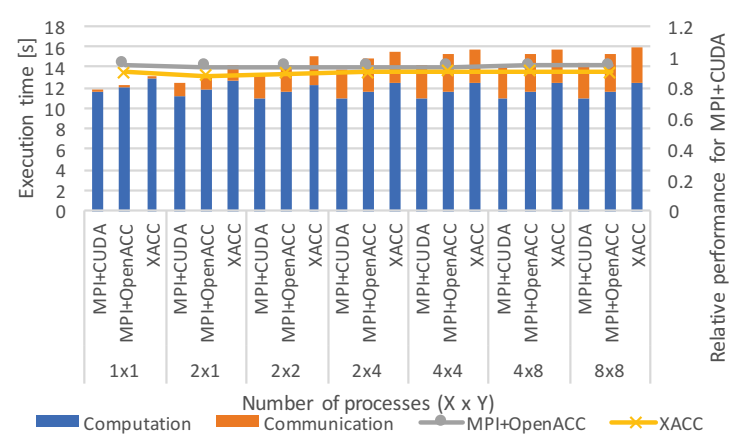

(a) Overall time

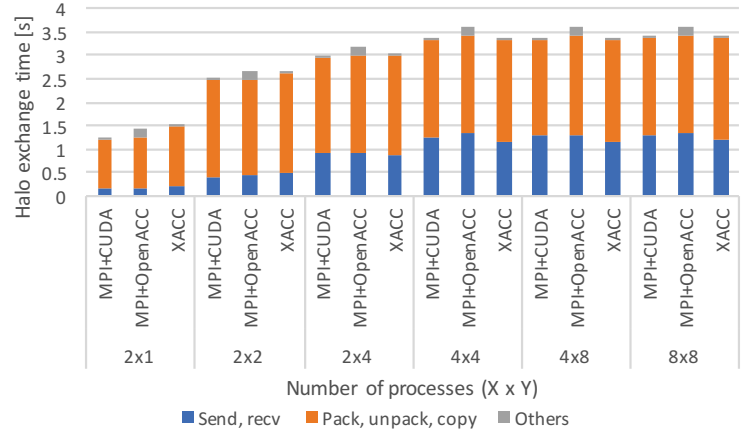

(b) Halo exchange time

Figure 14: Execution time for $960^{2}$ cells with weak scaling.

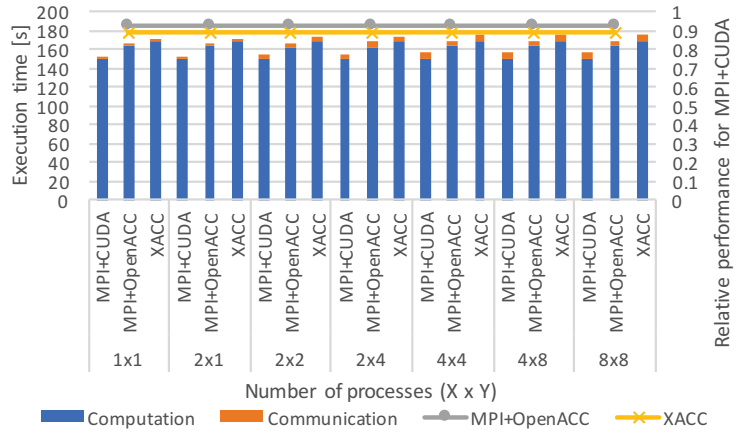

(a) Overall time

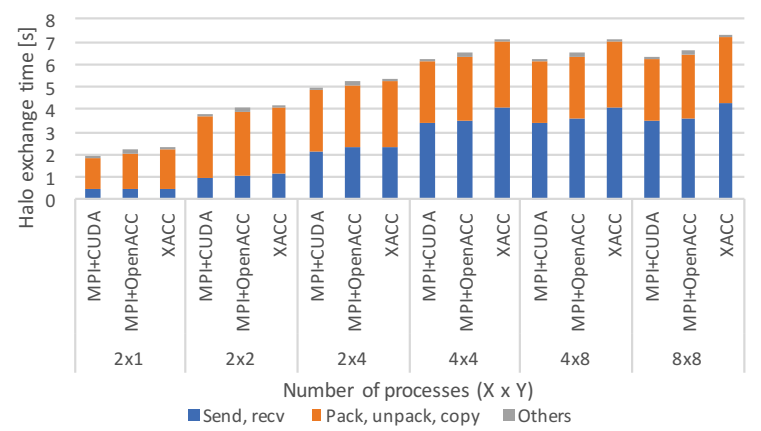

(b) Halo exchange time

Figure 15: Execution time for $3840^{2}$ cells with weak scaling.

directive-based description in XACC retained a better image of the serial version than MPI+OpenACC.
However, the XACC DSLOC without the addition of directive code was nearly 100 and still large. The detailed explanation for this difference is as follows. IF and ENDIF statements determining 


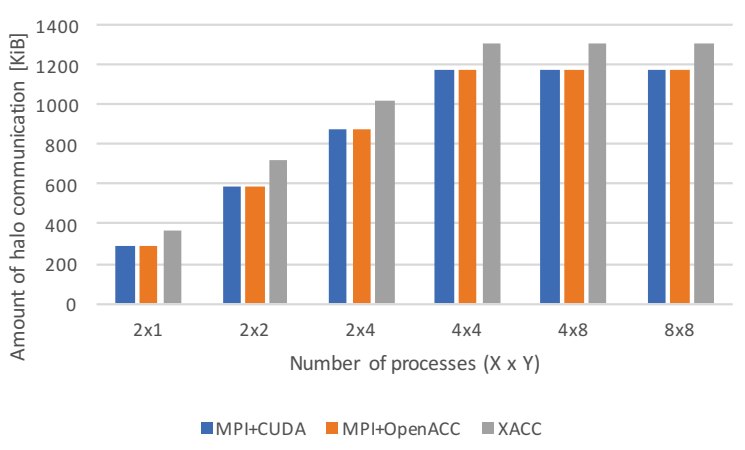

(a) $960^{2}$ cells

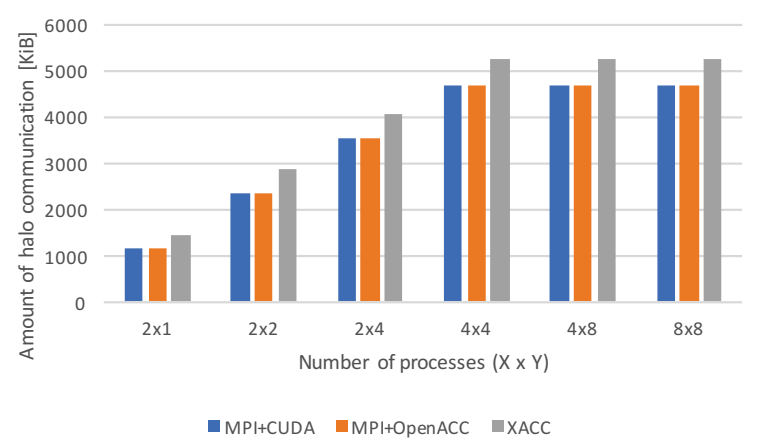

(b) $3840^{2}$ cells

Figure 16: Halo communication amount per iteration on each node with weak scaling.

whether to do the reflect directive or not added 30 lines (there are IF, ENDIF statements for each of the directives). USE statements for the module containing variables used in the XMP version added 15 lines. The codes related to gblock distribution added nine lines. The other differences in the codes were getting the assigned index range, and getting the node number and the number of nodes, etc.

\subsection{Discussion}

Our comparison of MPI+CUDA and MPI+OpenACC showed that OpenACC performed well considering the simple code description required. The comparison of MPI+OpenACC and XACC showed that the performance of XACC was slightly degraded but still adequate, although this was a minor issue considering the benefit of concise description using directives. In addition, the halo exchange time with XACC was shorter than that using MPI+OpenACC in some cases because the XACC communication runtime is written in MPI and CUDA, and well tuned. Due to the simplicity of programming and its well tuned communication, XACC has good usability for applications that are decomposed in the Cartesian domain and where the communication mainly comprises halo exchange, such as CloverLeaf. For applications with complex and undescribable decomposition and communication in the directives, the XACC local-view model's coarray feature in the accelerator memory allows us to describe complex communication with simple syntax. Moreover, combining the global-view and local-view models is possible in XACC, such as the XMP-hybrid view model[17]

As mentioned earlier, the XACC version still requires the addition of code and modifications compared with the serial version without directives and performance degradation may occur due to unnecessary updates in the halo exchange process. We consider that the following improvements may minimize these problems.

The first improvement involves the if clause in the XMP and XACC communication directives such as reflect and reduction. Figure 17 shows the syntax of the if clause. When an if clause appears in a communication directive, the program will conditionally communicate data. When the condition in the if clause is evaluated as .false., the directive does not allow communication. When the condition is evaluated as .true., the directive allows communication as if there is no if clause. This would be simpler than adding

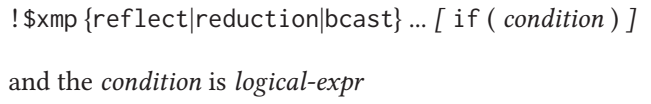

Figure 17: Syntax of the if clause.

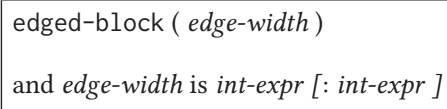

Figure 18: Syntax of the edged-block distribution method.

IF and ENDIF statements around the communication directive, and the IF statement has no statement if we assume that the directive is ignored.

The second improvement involves the edged-block distribution method for the distribute and template_fix directives. The syntax of the edged-block is shown in Figure 18. If one integerexpression is specified for the edge-width, then it is the width of both the lower and upper side edges. If two integer-expression is specified, the first is the lower side edge width and the second is the upper side edge width. The edged-block distributes the target template dimension in a block manner such that the dimension lacks the specified lower and upper edge indices. Next, the lower and upper edges are added to the lowermost and uppermost node's blocksize, respectively. For example, if template $t(1: 10)$ is distributed in an edged-block (1) distribution method onto nodes $p(4)$, then the template sizes of each node are $(/ 3,2,2,3 /)$. By using the edged-block distribution method, line 20 in Figure 5 can be described as “\$xmp template_fix (edged-block(2:3), edged-block(2:3))\&," and line 9 in Figure 8 can be rewritten as " $\$$ xmp template $t$ (edgedblock(2:3), edged-block(2:3)) onto p."

The third improvement involves the offset clause with the reflect directive, which specifies the offset when updating the shadow region. The syntax of the offset clause is shown in Figure 19. The syntax of the offset clause is similar to that of the 


$$
\begin{aligned}
& \text { !\$xmp reflect ... [ offset ( reflect-offset [,reflect-offset]... ) ] } \\
& \text { and reflect-offset is int-expr [:int-expr] }
\end{aligned}
$$

Figure 19: Syntax of the offset clause in the reflect directive

width clause. If one integer expression is specified, then it is offset for both the lower and upper side shadows. If two integer expressions are specified, then the first is the lower side offset and the second is the upper side offset. The reflect directive updates the width shadow elements with skipping offset shadow elements from the inside. If there is no off set clause, the directive is treated as if offset(0) is specified. For example, if a distributed array with shadow(4) is updated by the reflect directive with width(2) and offset(1), then the innermost and outermost shadow elements are not updated, and the middle two elements are updated. By using the offset clause, we can describe halo exchange for cell center quantity arrays as "\$xmp reflect(array-name) width(2) offset(0:1)" and the communication size will be the same as the MPI+CUDA and MPI+OpenACC versions.

\section{CONCLUSION}

A PGAS language XACC has been proposed that integrates the existing PGAS language XMP and an accelerator programming model OpenACC in order to improve the productivity of application development for accelerated clusters. XACC allows us to describe applications by adding directives to the serial version of codes for accelerator programming on a distributed-memory system. In this study, we implemented a hydrodynamics mini-application called CloverLeaf by using XACC, and we evaluated the performance and productivity of this method. This is the first XMP or XACC application to use a staggered grid arrangement. According to the evaluation, the performance level of the XACC version was $87-95 \%$ compared with that by the MPI+CUDA version and $93-101 \%$ of that by the MPI+OpenACC version with strong scaling, and $88-91 \%$ of that by the MPI+CUDA version and $94-97 \%$ of that by the MPI+OpenACC version with weak scaling. In particular, the halo exchange time with XACC was better than that with MPI+OpenACC in some cases because the Omni XACC runtime is written in MPI and CUDA, and it is well tuned. In terms of productivity, XACC performed better than MPI+CUDA and MPI+OpenACC because of the simple directive descriptions based on serial codes. XACC has high productivity and performance comparable to that of MPI+CUDA or MPI+OpenACC. We also proposed three XMP extensions to reduce the code modifications required in serial versions of codes and to improve the performance.

In our future research, we will implement and evaluate the proposed extensions. Moreover, we will evaluate CloverLeaf with GPU Direct or TCA features in order to achieve better performance, especially with strong scaling.

\section{ACKNOWLEDGMENTS}

This study was supported in part by the JST/CREST program entitled "Research and Development on Unified Environment of Accelerated Computing and Interconnection for Post-Petascale Era" in the research area of "Development System Software Technologies for post-Peta Scale High Performance Computing." This study used the computational resources of HA-PACS/TCA provided by the Interdisciplinary Computational Science Program of the Center for Computational Sciences, University of Tsukuba.

\section{REFERENCES}

[1] Center for Computational Sciences, University of Tsukuba. 2017. Supercomputers - Center for Computational Sciences. (2017). Retrieved August 11, 2017 from https://www.ccs.tsukuba.ac.jp/eng/supercomputers/\#HA-PACS

[2] T. Hanawa, Y. Kodama, T. Boku, and M. Sato. 2013. Interconnection Network for Tightly Coupled Accelerators Architecture. In High-Performance Interconnects (HOTI), 2013 IEEE 21st Annual Symposium on. 79-82.

[3] Francis H. Harlow and J. Eddie Welch. 1965. Numerical Calculation of TimeDependent Viscous Incompressible Flow of Fluid with Free Surface. The Physics of Fluids 8, 12 (1965), 2182-2189.

[4] J. A. Herdman, W. P. Gaudin, S. McIntosh-Smith, M. Boulton, D. A. Beckingsale, A. C. Mallinson, and S. A. Jarvis. 2012. Accelerating Hydrocodes with OpenACC, OpenCL and CUDA. In 2012 SC Companion: High Performance Computing, Networking Storage and Analysis. 465-471.

[5] Charles H. Koelbel and Mary E. Zosel. 1993. The High Performance FORTRAN Handbook. MIT Press, Cambridge, MA, USA.

[6] A. C. Mallinson, David A. Beckingsale, W. P. Gaudin, J. A. Herdman, and Stephen A. Jarvis. 2013. Towards Portable Performance for Explicit Hydrodynamics Codes. In 1st International Workshop on OpenCL (IWOCL 13).

[7] A. C. Mallinson, S. A. Jarvis, W. P. Gaudin, and J. A. Herdman. 2014. Experiences at Scale with PGAS Versions of a Hydrodynamics Application. In Proceedings of the 8th International Conference on Partitioned Global Address Space Programming Models (PGAS '14). ACM, New York, NY, USA, Article 9, 11 pages.

[8] Mantevo.org. 2017. Home of the Mantevo Project. (2017). Retrieved August 11, 2017 from https://mantevo.org/

[9] Masahiro Nakao, Hitoshi Murai, Hidetoshi Iwashita, Akihiro Tabuchi, Taisuke Boku, and Mitsuhisa Sato. 2017. Productivity and Performance of XcalableACC Language for Acceralated Clusters. (Feb 2017). Poster presented at The 7th AICS International Symposium.

[10] Masahiro Nakao, Hitoshi Murai, Takenori Shimosaka, Akihiro Tabuchi, Toshihiro Hanawa, Yuetsu Kodama, Taisuke Boku, and Mitsuhisa Sato. 2014. XcalableACC: Extension of XcalableMP PGAS Language Using OpenACC for Accelerator Clusters. In Proceedings of the First Workshop on Accelerator Programming Using Directives (WACCPD '14). 27-36.

[11] OpenACC-standard.org. 2017. OpenACC. (2017). Retrieved August 11, 2017 from http://www.openacc.org

[12] PC Cluster Consortium. 2017. PC Cluster Consortium. (2017). Retrieved August 11, 2017 from http://www.pccluster.org

[13] John Reid. 2010. Coarrays in the next Fortran Standard. ISO/IEC fTC1/SC22/WG5 N1824 (Apr. 2010).

[14] RIKEN AICS and University of Tsukuba. 2017. Omni Compiler Project. (2017). Retrieved August 11, 2017 from http://omni-compiler.org

[15] RIKEN AICS and University of Tsukuba. 2017. XcalableACC Launguage Specification Version 1.0. (2017). Retrieved August 11, 2017 from http://xcalablemp.org/download/XACC/xacc-spec-1.0.pdf

[16] Akihiro Tabuchi, Masahiro Nakao, Hitoshi Murai, Taisuke Boku, and Mitsuhisa Sato. 2017. Implementation and Evaluation of One-sided PGAS Communication in XcalableACC for Accelerated Clusters. In Proceedings of the 17th IEEE/ACM International Symposium on Cluster, Cloud and Grid Computing (CCGrid '17). 625634.

[17] Keisuke Tsugane, Taisuke Boku, Hitoshi Murai, Mitsuhisa Sato, William Tang, and Bei Wang. 2016. Hybrid-view programming of nuclear fusion simulation code in the PGAS parallel programming language XcalableMP. Parallel Comput. 57 (2016), 37-51. https://doi.org/10.1016/j.parco.2016.05.016

[18] UK Mini-App Consortium. 2017. UK Mini-App Consortium. (2017). Retrieved October 27, 2017 from http://uk-mac.github.io/

[19] XcalableMP Specification Working Group. 2017. XcalableMP WebSite. (2017) Retrieved August 11, 2017 from http://www.xcalablemp.org 